\title{
Effect of Anhydride Grafting Agent on Trap Levels of Low- Density Polyethylene
}

\author{
Lijuan $\mathrm{He}^{1,2}$, Zhenhua Yuan ${ }^{1}$, Xiong Yang ${ }^{1}$, Huiqing Niu ${ }^{1}$, Chao Zhu ${ }^{1}$, \\ Dawei $\mathrm{Li}^{1}$, Lei Zhao ${ }^{1}$ and Chuntian Chen ${ }^{1}$ \\ 1Harbin University of Science and Technology, Harbin, 150080, P.R.China \\ 2 Key Laboratory of Engineering Dielectric and Its Application, Ministry of \\ Education, Harbin University of Science and Technology, 150080, China \\ chenchuntian730@sohu.com
}

\begin{abstract}
Trap distribution of the low-density polyethylene(LDPE) modified by anhydride grafting agent was studied and the effect of grafting agent on the trap levels was analyzed by the method of Photo-stimulated Discharge in this paper. Different concentrations and types of same concentration of LDPE modified by anhydride grafting agent were measured by using continuous UV scanning. The results showed that different concentrations and types of grafting agent had effect on trap levels distribution of LDPE. The trap depth would decrease with the anhydride grafting agent content increasing and increase with its damaged condition increasing when anhydride grafting agent was destroyed. Anhydride grafting agent are widely applied to the production and research of high pressure insulating polyethylene materials. The purpose of this paper is to elaborate the effect of anhydride grafting agent on the insulating properties of polyethylene material and explain the strongest binding capacity of anhydride grafting agent to space charge.
\end{abstract}

Keywords: Photo-stimulated Discharge; Anhydride; Trap level; Chemical Structure

\section{Introduction}

The study of Space charge in polymer dielectric has been received more and more attention by relative researchers. The electric fields of dielectric are more easily distorted because of space charge[1], which can affect the breakdown characteristic of dielectric. The breakdown even aging of polymer dielectric induced by the space charge will lead to the termination of its insulation life when high-pressure insutating material work in high voltage environment for a long time. Therefore, many characteristics of the polymer dielectric are closely related to the distribution of space charge[2-5]. The main causes of the aging of the high-pressure insutating material can be divided into internal and external causes. The internal cause is that other chemicals or chemical structure can be introduced into high-pressure insutating material during the production process. The external cause is that the external strains would make high-pressure insutating material produced defect during working. This paper explained the impact of their chemical structure of the insulating properties by simulating the effect of the underlying reasons on the trap levels of LDPE using modified Low-Density Polyethylene. In order to furtuher illustrated the relationship between the chemical structure and the space charge, this paper simulated external factors that Low-Density Polyethylene may encounter destruction external factors during its working by destroying chemical structure of above anhydride grafting agent. 


\section{Preparation of Samples}

\subsection{Experimental Equipment}

Photo-stimulated Discharge equipments: including laser (the type of excitation light source: NT342A-SH), test sample chamber, energy meter and electrometer. Infrared spectroscopy(BRUKER Equinox55): made by Brukeroptics. Platen press $(\mathrm{XLB}-\mathrm{D} / \mathrm{Q} 350 \times 350)$ : Nominal clamping force of $0.60 \mathrm{MN}$, the specifications of heat shield of $350 \times 350 \mathrm{~mm}$, hot plate spacing of $150 \mathrm{~mm}$, working layers of two layers, motor power of $2.2 \mathrm{~kW}$, made by Huzhou Shuangli Automation Equipment Co., Ltd.

\subsection{Raw Materials}

Benzoyl peroxide, AR, made by the sixth Tianjin Chemical Reagent industry; Hydrogen peroxide, the mass fraction of $30 \%$, made by Tianjin Zhiyuan company; Maleic anhydride, > 99.6\%, made by Tianjin jiexing Ltd; Xylene, AR, made by Tianjin Jiayu Ltd; Ethanol, made by Tianjin Jiayu Ltd.; Dimethicone, made by Tianjin Jiayu Ltd.

\subsection{The Sample Proportion and Serial Number}

Different proportion and types of all samples were shown in table 1:

Table 1.Proportion and Number of Samples

\begin{tabular}{lll}
\hline Number & Name & Mass ratio \\
\hline C & LDPE & $0:$ Any value \\
G6-1 & LDPE modified by maleic anhydride & $1: 6$ \\
G4-1 & LDPE modified by maleic anhydride & $1: 4$ \\
G3-1 & LDPE modified by maleic anhydride & $1: 3$ \\
S6-1 & After hydrolysis LDPE modified by maleic anhydride & $1: 6$ \\
S4-1 & After hydrolysis LDPE modified by maleic anhydride & $1: 4$ \\
S3-1 & After hydrolysis LDPE modified by maleic anhydride & $1: 3$ \\
\hline
\end{tabular}

\section{Experimental and Theory}

We stimulated three phases to show the change of insulation property. Phase one illustrated production process of modified polyethylene. Phase two illustrated the process that Polyethylene affected by external condition produced defect. Phase three was the testing process.

\subsection{Stimulation of Production Process}

Firstly, installing experimental device, a condenser, three-necked flask, two water-cooled tube, a thermometer, stopper sub, heating mantle, a magnetic .Then $200 \mathrm{ml}$ xylene, a certain amount of LDPE and Maleic anhydride were mixed sequentially in this order, and stirred after that. The reaction system was heated with dimethyl silanes, gradually increasing the temperature. The experimental results were recorded for every ten degrees rising. When the temperature reached 80 degrees, slowly heated. Benzoyl peroxide was added when LDPE completely dissolved,then increased the temperature and counted reaction time until benzoyl peroxide completely dissolved. After the completion of the reaction, the reaction was slowly poured into absolute ethanol, soaked, filtered, and washed. Prepared product was dried in a vacuum oven having four samples reserved. 


\subsection{Stimulation of Use Process}

Firstly, Mounting experimental device, thermostat electric stirrer, reflux condenser, dropping funnel and three-necked flask . different proportions LDPE modified in phase was placed into three-necked flask. Then a certain amount of distilled water and catalyst were added into three-necked flask. Heat the sample until completely dissolved, continue to heat until 96 degrees. A certain amount of initiator Hydrogen peroxide was dropwise added into three-necked flask and stirred. Keep the temperature for $2 \mathrm{~h}$ after completion of titration. Then heating was stopped, the orange solution was placed on a glass plate and was dried, obtaining other three sets of samples.

\subsection{Testing Process}

A small amount of the samples weighted in Phase one and Phase two was pressed on vulcanizing machine. The temperature of vulcanizing machine is 95 degrees; Pressing time 15 minutes; pressure 10Mpa. And then thickness of $120 \mathrm{um}$ to $170 \mathrm{um}$ and diameter of $10 \mathrm{~cm}$ were obtained in seven groups of sample film. The translucent gold electrode was steamed on the two surfaces of the seven groups of film. And the thickness of gold electrode was 50nm; charging voltage between $4000 \mathrm{~V}$ to $4500 \mathrm{~V}$; charging time $15 \mathrm{~min}$. After charging, the seven groups of sample were shorted circuit for $0.5 \mathrm{~h}$ to make internal trapped charge keep stable before the photonstimulated discharge experiment begining.

\subsection{Theory and System of Photon-Stimulated Discharge}

In this method, the sample that is injected discharge (using the method of Corona, electric field, light) is irradiated by luminescence, its wavelength could continuous change. Trapped charges are released by the excitation of mono-energy photons, therefore, current released by electric charge formed the external circuit. Trap energy distribution can be obtained by analyzing the relationship between PSD current and wavelength luminescence.

The testing system of PSD was made up of excitation source, the test sample chamber, the energy meter, static meter and data acquisition system. Figure 1 shows the PSD measuring system.

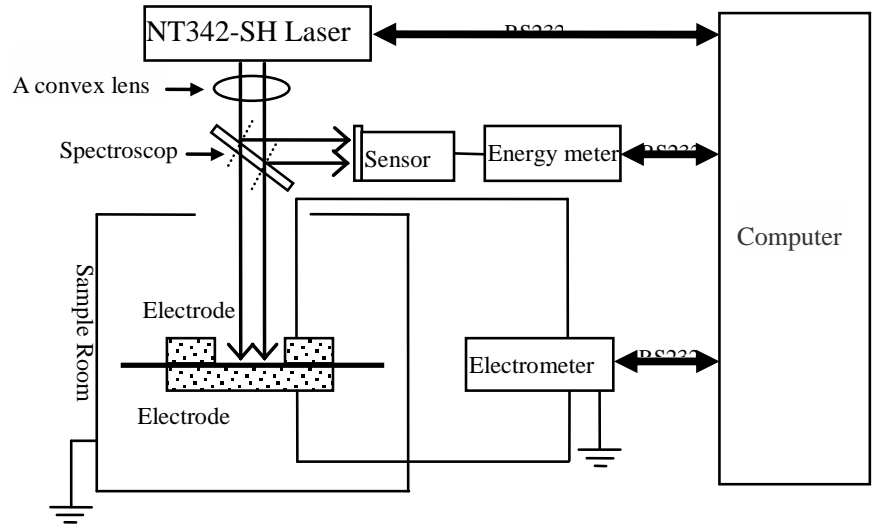

Figure 1.Experiment Principle of the PSD Test System Diagram

\section{Results and Discussion}




\subsection{Infrared Spectroscopy}

In order to distinguish the synthesized products, seven groups of sample were analyzed by Infrared Spectroscopy. Since samples contained unreacted maleic anhydride, synthesized products were washed with acetone for several times, dried , and then made into $15 \mathrm{~mm} * 15 \mathrm{~mm}$ samples for the analysis of Infrared Spectroscopy . Figure 2 showed Infrared Spectroscopy of mixed three samples . The first sample was LDPE unmodified, the red curve in Figure 2. The second sample was LDPE modified by maleic anhydride, the black one in Figure 2. The third sample was the product of hydrolysis of LDPE modified by maleic anhydride, the blue one in Figure 2.

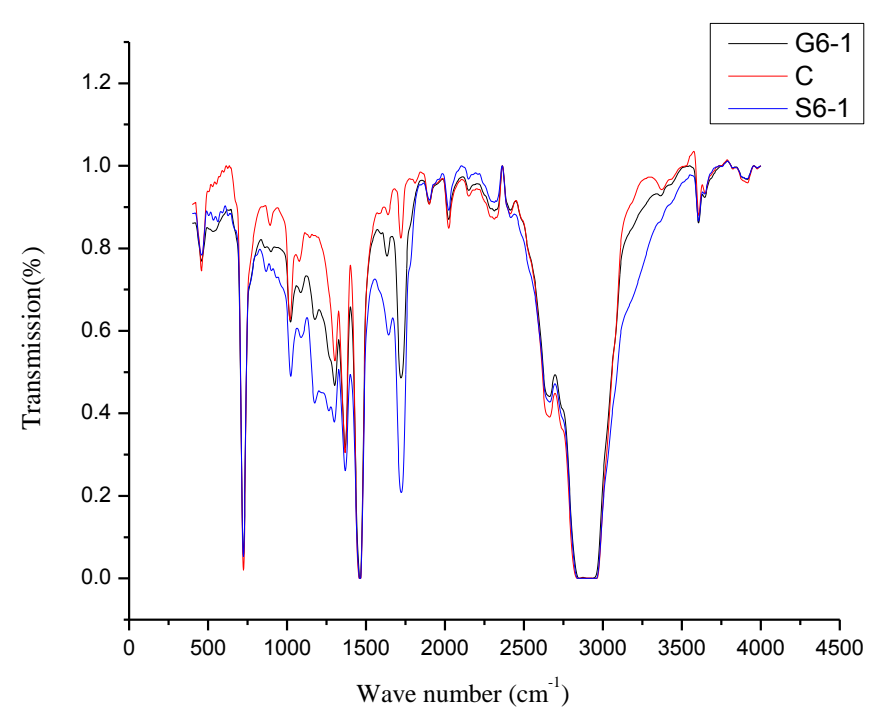

Figure 2.IR Spectra of Three Samples

From the Figure 2 , there was characteristic peak in $1460 \mathrm{~cm}-1$ region. There was strong characteristic peaks of cyclic anhydrides in $1620 \mathrm{~cm}-1$ to $1720 \mathrm{~cm}-1$ region. There was characteristic peaks of anhydrides of five-member-ring in $1790 \mathrm{~cm}-1$ to $1800 \mathrm{~cm}$-1region .There was C-O Stretching vibration peak of cyclic anhydrides in $1150 \mathrm{~cm}-1$ to $1250 \mathrm{~cm}-1$ region and $\mathrm{C}=\mathrm{C}$ characteristic absorption peaks of cyclic anhydrides in $1030 \mathrm{~cm}-1$ region. There was no characteristic peaks of Vinyl in $960 \mathrm{~cm}-1$ region in all of three samples, proving that three samples were not crosslinked. Therefore only considering LDPE modified by maleic anhydride, there was strong five-membered ring conjugate peak in $1570 \mathrm{~cm}-1$ to $1670 \mathrm{~cm}-1$ region when weight ratio of polyethylene and maleic anhydride was $4: 1$.Above situation proved that maleic anhydride excessed. When weight ratio of polyethylene and maleic anhydride was $6: 1$, there was not above peak, proving that maleic anhydride monomer did not exist.

Author names and affiliations are to be centered beneath the title and printed in Times New Roman 12-point, non-boldface type. Multiple authors may be shown in a two or three-column format, with their affiliations below their respective names. Affiliations are centered below each author name, italicized, not bold. Include email addresses if possible. Follow the author information by two blank lines before main text.

\subsection{Results Analysis of PSD Continuous scan of LDPE Modified}


The current of PSD was affected by samples, samples electrode, charging conditions and ambient. In order to describe the relative intensity of PSD better as well as get the trap energy distribution of each sample, energy normalization was applied to process current spectrum of seven groups of samples. Energy normalization meant all points on the curve divided by the corresponding to the energy values in order to eliminate the impact of uneven irradiation and eliminate interference of uneven energy, finally per unit of PSD current was obtained, shown in Figure 3, which was normalized energy spectra of LDPE.

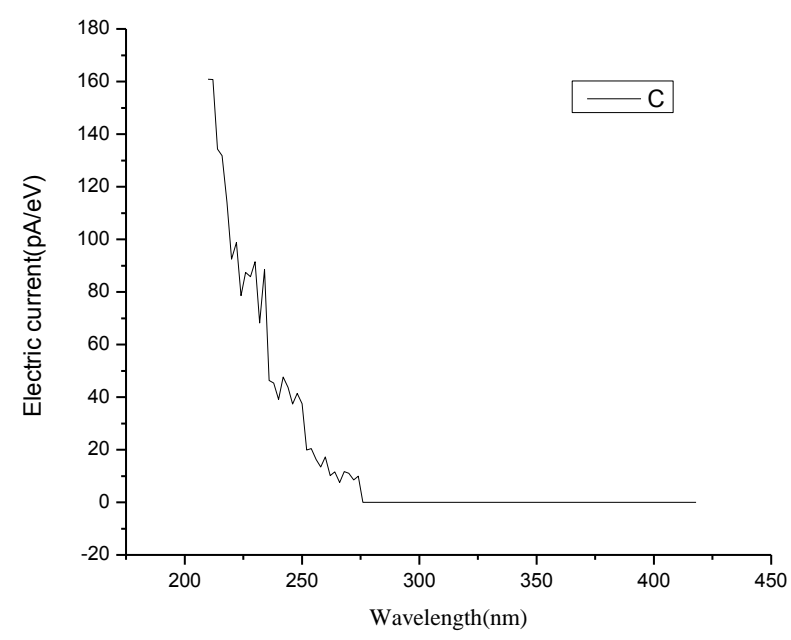

Figure 3.Energy Spectra of LDPE Unmodified after Normalization

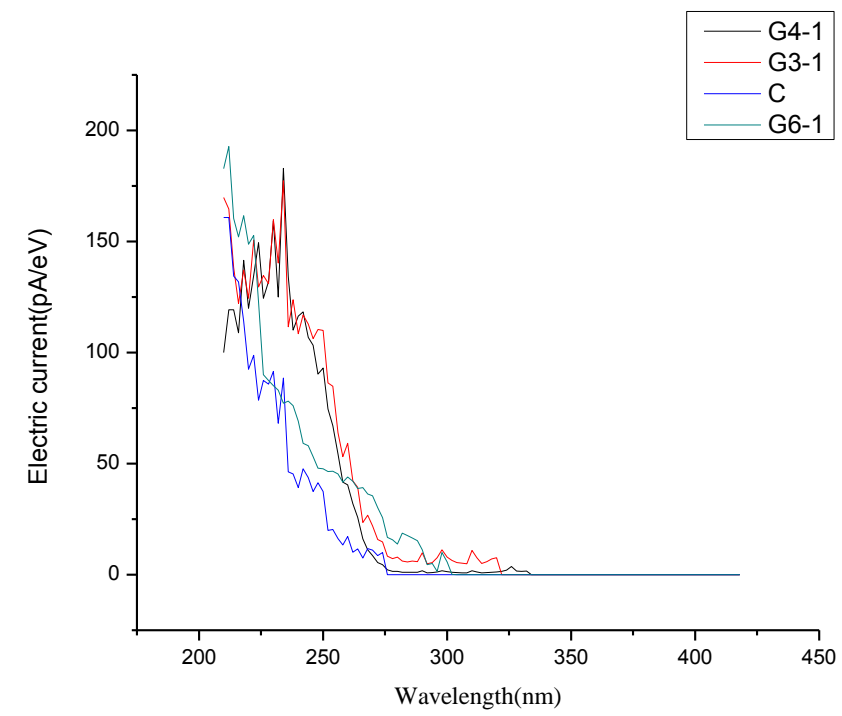

Figure 4.Comparative Spectra between the Standard Sample and the Sample Modified by Maleic Anhydride

The Figure 4 showed that PSD spectrum of LDPE modified and PSD spectrum of LDPE unmodified. Initial waves were the same at about $300 \mathrm{~nm}$. However, peak was not the same as the corresponding current wavelength, wavelength of LDPE unmodified was less than $220 \mathrm{~nm}$. wavelength of LDPE modified was between $220 \mathrm{~nm}$ and $250 \mathrm{~nm}$ when the wavelength increased with the maleic anhydride content 
increasing. It can be proved that maleic anhydride grafting agent of LDPE modified would make the trap light significantly.

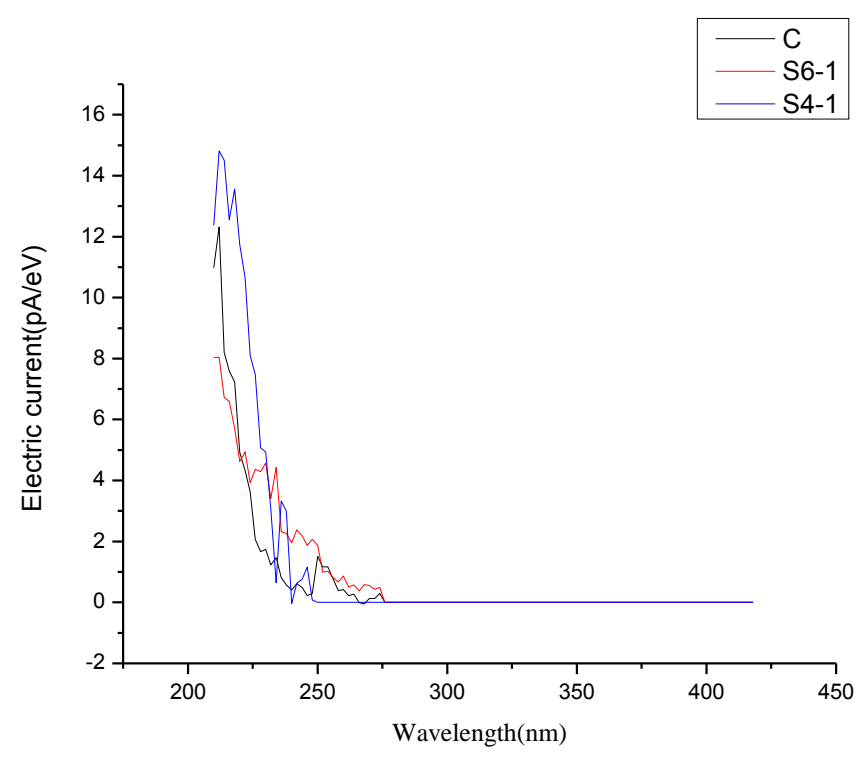

Figure 5.Comparative Spectra between the Standard Sample and the Poduct
after the Destruction of Maleic Anhydride

The Figure 5 showed that the initial waves of Hydrolyzed polyvinyl modified by maleic anhydride was shorter than that of LDPE unmodified, both of them at about $250 \mathrm{~nm}$. However, their current wavelength of corresponding peak were less than $220 \mathrm{~nm}$.As is shown that the trap of hydrolyzed polyvinyl modified by maleic anhydride was deeper than LDPE unmodified in the Figure 5.

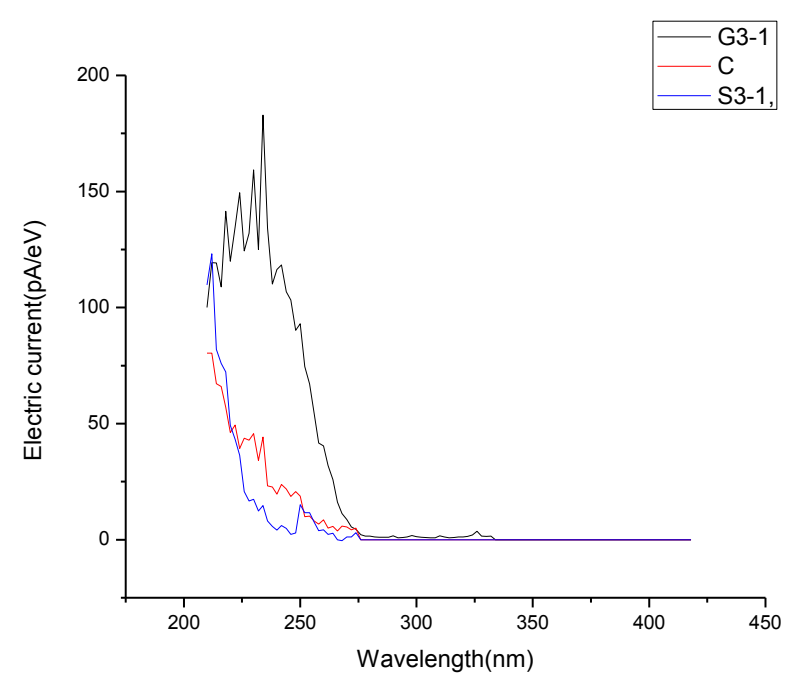

Figure 6. Analog Atlas of Three Samples

The Figure 6 showed that the current wavelength of LDPE modified by maleic anhydride was bigger than the LDPE unmodified and the current wavelength of Hydrolyzed polyvinyl modified maleic anhydride was the shortest, that is, the deepest trap. One of reasons may be that Hydrolyzed anhydride produced ester during forming the film, increasing the mesh structure of this material. 


\section{Conclusion}

This experiment studied the effect of different kinds and different matching of anhydride grafting agent on trap levels of LDPE by the method of PSD continuous scan. This method was applied to simulate the production process of LDPE and defect process when LDPE was affected by external condition. The results showed that the depth of trap would decrease when content of maleic anhydride was between 1:6 and 1:3. While the content of damaged maleic anhydride was between 1:6 and 1:3, the depth of trap would increase. With the change of trap, the life of the insulating material would be affected.

\section{Acknowledgements}

This work has been supported by the Heilongjiang province Natural Science Foundation of China under Contract No.E201220.

\section{References}

[1] T. Tanaka. Buds for treeing in epoxy nanocomposites and their possible interaction with nano fillers. IEEE International Conference on Solid Dielectrics(2010); July 4-9, Potsdam, Germany.

[2] T. A. L. Burgo, C. A. Rezende and S. Bertazzo. Electric potential decay on polyethylene: Role of atmospheric water on electric charge build-up and dissipation. Journal of Electrostatics. 69(2011), pp.401-409.

[3] T. J. Lewis. Interfaces are the Dominant Feature of Dielectrics at the Nanometric Level. IEEE Transactions on Dielectrics and Electrical Insulation. 11(2004), pp. 739-853.

[4] Q. Q. Lei and Y. Fan, Effect of Inorgamic Filler on Thermally Stimulated Current in Low-density Polyethylene. Materials Sci. \& Technol(1997). 13 , pp. 223-226.

[5] Z. E. Zhe and Y. W. Zhang. Study on Charge Trap Distribution in LLDPE by Photo-Stimulated Discharge Current. IEEE International Conference on Properties and Applications of Dielectric Materials(2009); July 19-23, Harbin, China.

[6] C. Kim, J. Z. Jian, J. Pingkai and X. Y. Huang. Investigation of Dielectric Behavior of Thermally Aged XLPE Cable in the High Frequency Rang. Polymer Testing(2006), 25, pp. 553-561.

[7] P. H. Zhang, Y. Fan and F. C. Wang. Conduction current characteristics and carrier mobility of both original and corona-resistant polyimide films, Chinese Physcis Letters(2005). 22 , pp.1253-1255.

[8] F. Q. Tian, Q. Q. Lei, X. Wang and Y. Wang. Effect of deep trapping states on space charge suppression in polyethylene/ZnO nanocomposite. Applied Physics Letters (2011). 99, pp. 142903. 
International Journal of Security and Its Applications

Vol. 10, No. 5 (2016) 